\title{
OBSERVATIONS ON LEPIDOPTERAN LEAF-SHELTERS AS MOLTING REFUGES FOR THE STINK BUG ELASMUCHA PUTONI (HETEROPTERA: ACANTHOSOMATIDAE)
}

\author{
BY SHIN-ICHI KUdO \\ Laboratory of Applied Zoology \\ Faculty of Agriculture \\ Hokkaido University \\ Sapporo, 060 Japan
}

\begin{abstract}
When molting to the adult, the acanthosomatid bug Elasmucha putoni form aggregations on the wild mulberry Morus bombycis. The 5th-instar nymphs and/or newly molted adults were found more often in leaves rolled, folded, or tied by tortricid larvae than on unmodified leaves. Bugs probably seek protection in lepidopteran leaf-shelters during molting.
\end{abstract}

\section{INTRODUCTION}

Many lepidopteran species build shelters by rolling, folding or tying leaves together with silk. Such leaf-shelters have been shown to provide a variety of benefits to herbivores (e.g., Damman, 1987; Sagers, 1992; Cappuccino, 1993). Herbivores other than sheltermakers, such as lepidopteran species which cannot build shelters of their own, homopterans, beetles and sawflies, also have been found in leaf-shelters (Carroll \& Kearby, 1978; Carroll et al., 1979; Cappuccino, 1993).

In the present report, I describe the use of lepidopteran leafshelters as adult-molting sites by the bug Elasmucha putoni Scott (Acanthosomatidae). This bug is a subsocial species: parent females guard their eggs and nymphs on the wild mulberry Morus bombycis Koizumi (Tachikawa, 1991). Nymphs live in aggregations and feed on the host fruit in the daytime. Nymphal feeding aggregations become smaller with progress of the instar, and in the final 5th instar, nymphs often feed solitarily (Kudo, unpublished).

Manuscript received 17 May 1994. 


\section{MATERIALS AND MethodS}

Field research was conducted in Misumai, Sapporo, on the island of Hokkaido in northern Japan in July 1991. Adult-molting aggregations or individuals of E. putoni, including 5th-instar nymphs molting or just before molting, and/or newly molted adults on the host tree, were examined. Rolled, folded, or tied leaves were opened and bugs (and other arthropods) contained within them were counted. The number of individuals per aggregation on unmodified leaves was also recorded. To learn the frequency of leaf-shelters, I randomly chose 30 branches with 10 to 20 shoots and counted all shelters and unmodified leaves.

\section{RESULTS AND DISCUSSION}

A total of 98 adult-molting aggregations or solitary bugs were found on mulberry trees, 63 of them in leaf-shelters and 35 on unmodified leaves. Of 73 leaf-shelters examined, 86.3\% ( $n=63)$ contained at least one bug (Fig. 1). Some bugs were present on the outside surface as well as the inside surface of the shelters. Leafshelters were rare on mulberry: only $4.3 \%$ of 1142 leaves examined were modified. The proportion of adult-molting aggregations (or individuals) in leaf-shelters to total aggregations $(64.3 \%, \mathrm{n}=98)$ was apparently larger than that of shelters to total leaves (chisquare $=388.1, \mathrm{p}<0.0001$ ), indicating that bugs seek shelter during the molting process. New adults left the shelters within one or two days after fully tanning. They stayed on the mulberry for a few days and then disappeared: they move to and breed on different plant species (Kudo, unpublished).

Most of the shelters were built by the lepidopterans, Olethreutes mori Matsumura or $O$. morivorum Matsumura (Tortricidae). At the time of examination, no larvae or pupae existed in any of the lepidopteran shelters, although pupal exuviae left were found in some shelters. Two of the shelters examined were constructed by spiders: one without bugs contained a spider with an egg sac, and another without a spider contained four bugs. Three shelters contained single earwigs. Figure 2 shows frequency distributions for bugs per unmodified leaf and per leaf-shelter. There was no significant difference in nymphal group size between the types of leaves (MannWhitney $\mathrm{U}$ test, $\mathrm{p}=0.645$ ). 


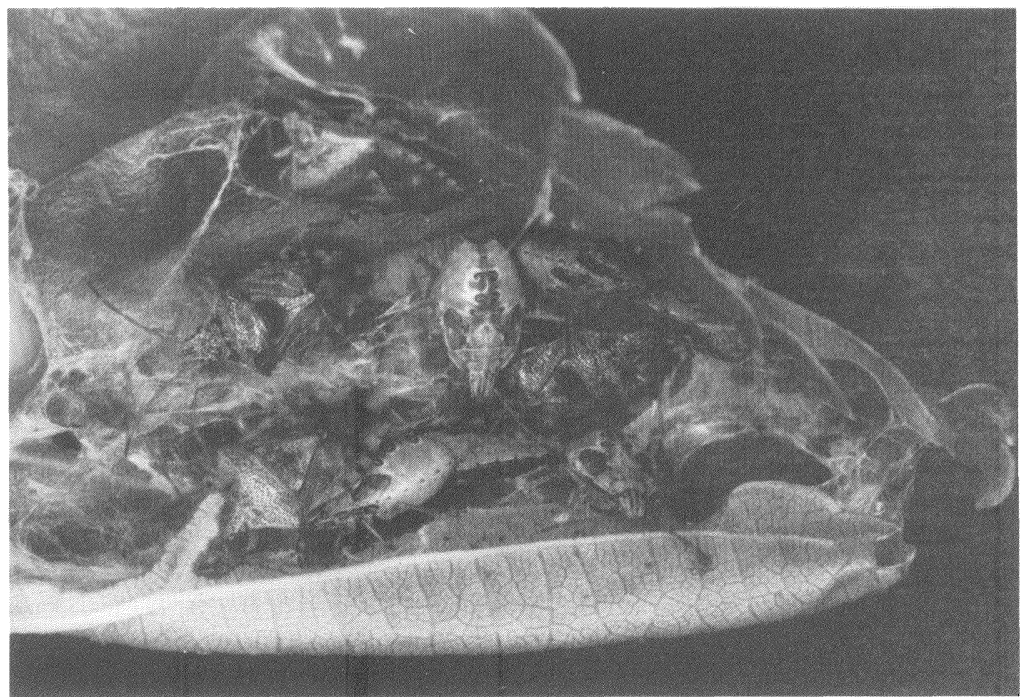

Fig. 1. An adult-molting aggregation of Elasmucha putoni within an open leafshelter.

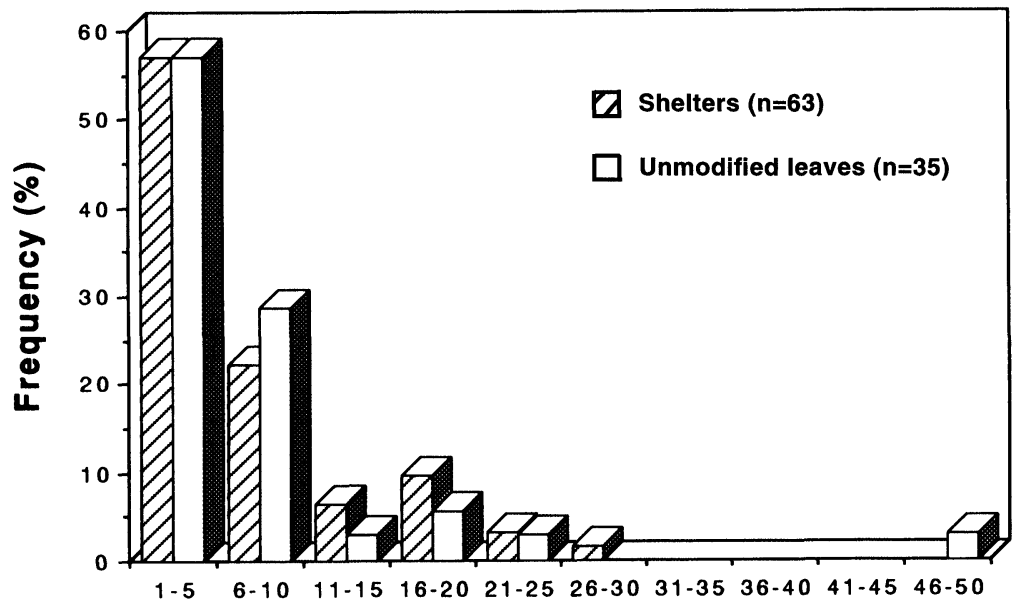

No. individuals/shelter or leaf

Fig. 2. Comparison of nymphal group size on unmodified leaves and in leafshelters. 
Living in a leaf-shelter may provide various benefits for hervivores, i.e., improved microhabitat or leaf quality (Hunter \& Willmer, 1989; Sagers, 1992), and refuge from predators (Damman, 1987; Cappuccino, 1993). Insects that utilize a pre-existing shelter may enjoy these benefits. In E. putoni, nymphs feed on the fruit of its host (Kudo, unpublished), and thus, gain no nutritional benefits while inhabiting leaf-shelters. On the other hand, molting and newly molted bugs with little mobility and soft cuticles are vulnerable to predators and harsh weather. Nabid bugs and thomisid and salticid spiders were sometimes observed to prey on late-instar nymphs or adults of E. putoni. It is very likely that leaf-shelters formed by tortricids provide molting and newly molted bugs protection from predators or harsh weather. The presence of sheltermaking lepidopterans, therefore, may indirectly increase bug survival.

\section{ACKNOWLEDGMENTS}

I thank N. Kuhara for help with field work and T. Kumata for identification of the moths. Anonymous referee's comments improved the manuscript. This study was supported in part by a JSPS Fellowship for Japanese Junior Scientists, and by a Grant-inAid for Scientific Research from the Ministry of Education, Science and Culture, Japan.

\section{REFERENCES}

Cappuccino, N. 1993. Mutual use of leaf-shelters by lepidopteran larvae on paper birch. Ecol. Entomol. 18: 287-292.

Carroll, M. R., and Kearby, W. H. 1978. Microlepidopterous oak leaftiers (Lepidoptera:Gelechioidea) in central Missouri. J. Kansas Entomol. Soc. 51: 457-471.

Carroll, M. R.; Wooster, M. T. Kearby, W. H., and Allen, D. C. 1979. Biological observations on three oak leaftiers: Psilocorsis quercicella, $P$. reflexella and $P$. cryptolechiella in Massachusetts and Missouri. Ann. Entomol. Soc. Am. 72: 441-447.

Damman, H. 1987. Leaf quality and enemy avoidance by the larvae of a pyralid moth. Ecology 68: 88-97.

Hunter, M. D., and Willmer, P. G. 1989. The potential for interspecific competition between two abundant defoliators on oak: leaf damage and habitat quality. Ecol. Entomol. 14: 267-277.

Sagers, C. L. 1992. Manipulation of host plant quality: herbivores keep leaves in the dark. Funct. Ecol. 6: 741-743.

Tachikawa, S. 1991. Studies on Subsocialities of Heteroptera in Japan. Tokyo Agricultural University Press, Tokyo. 167pp. (In Japanese) 

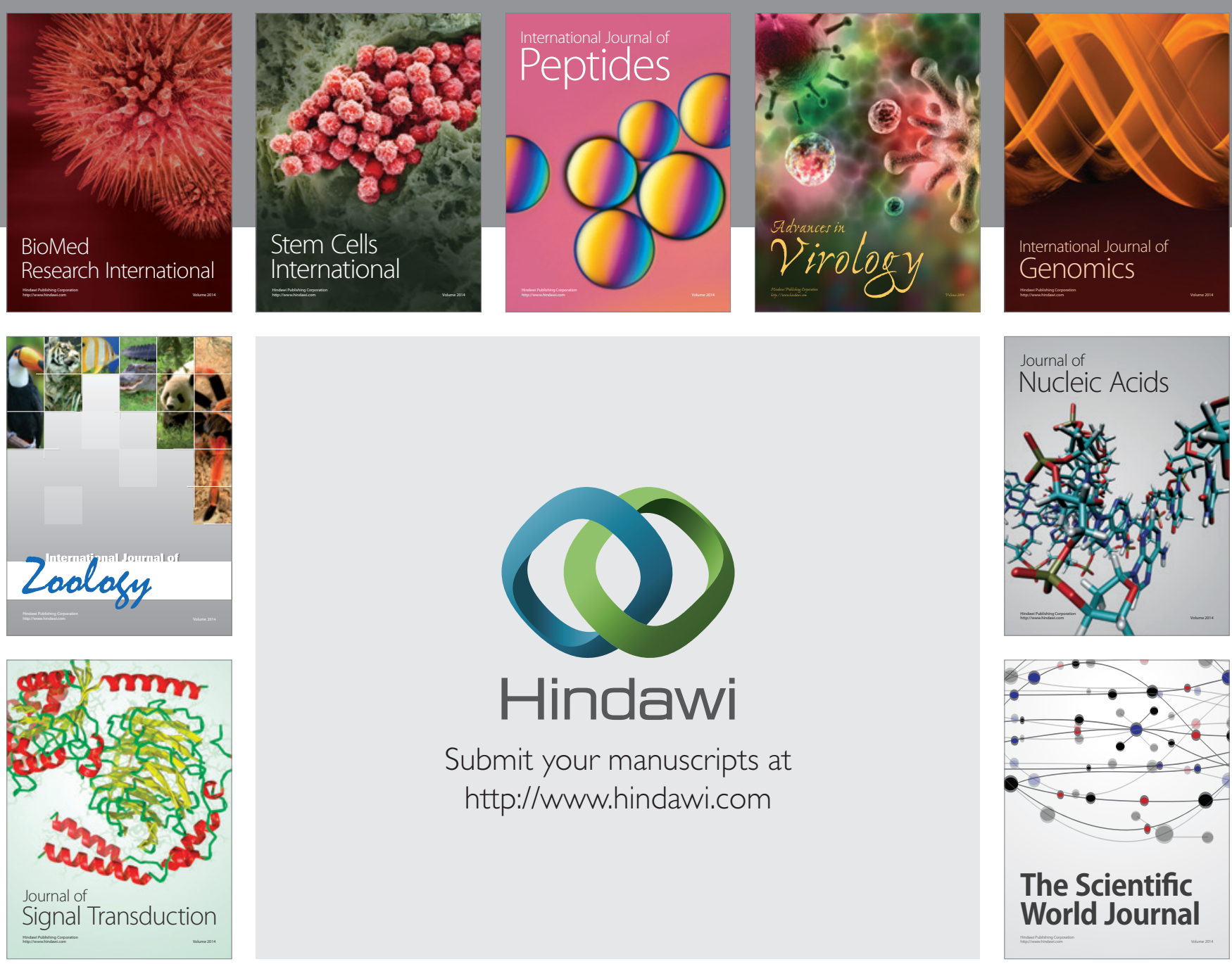

Submit your manuscripts at

http://www.hindawi.com
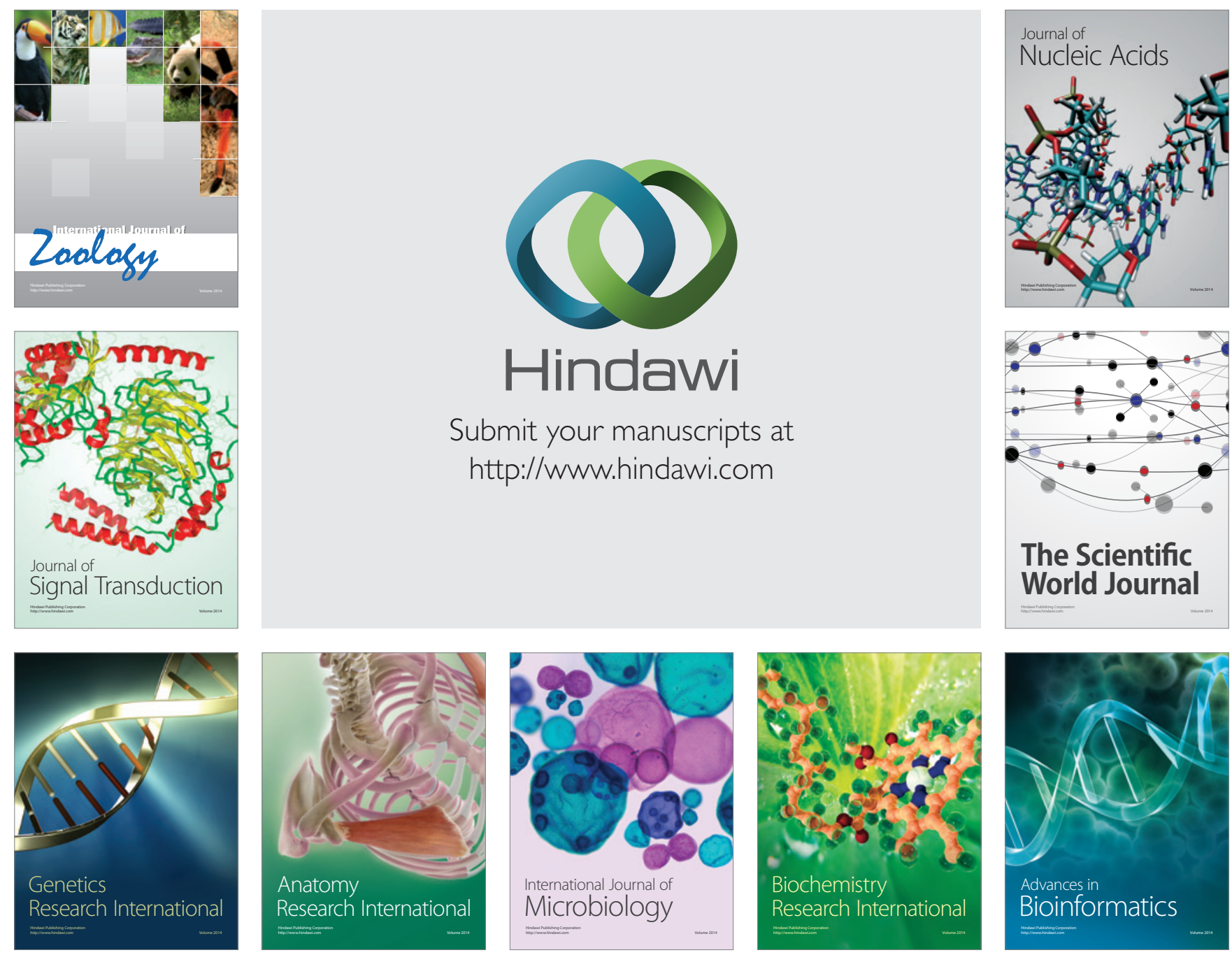

The Scientific World Journal
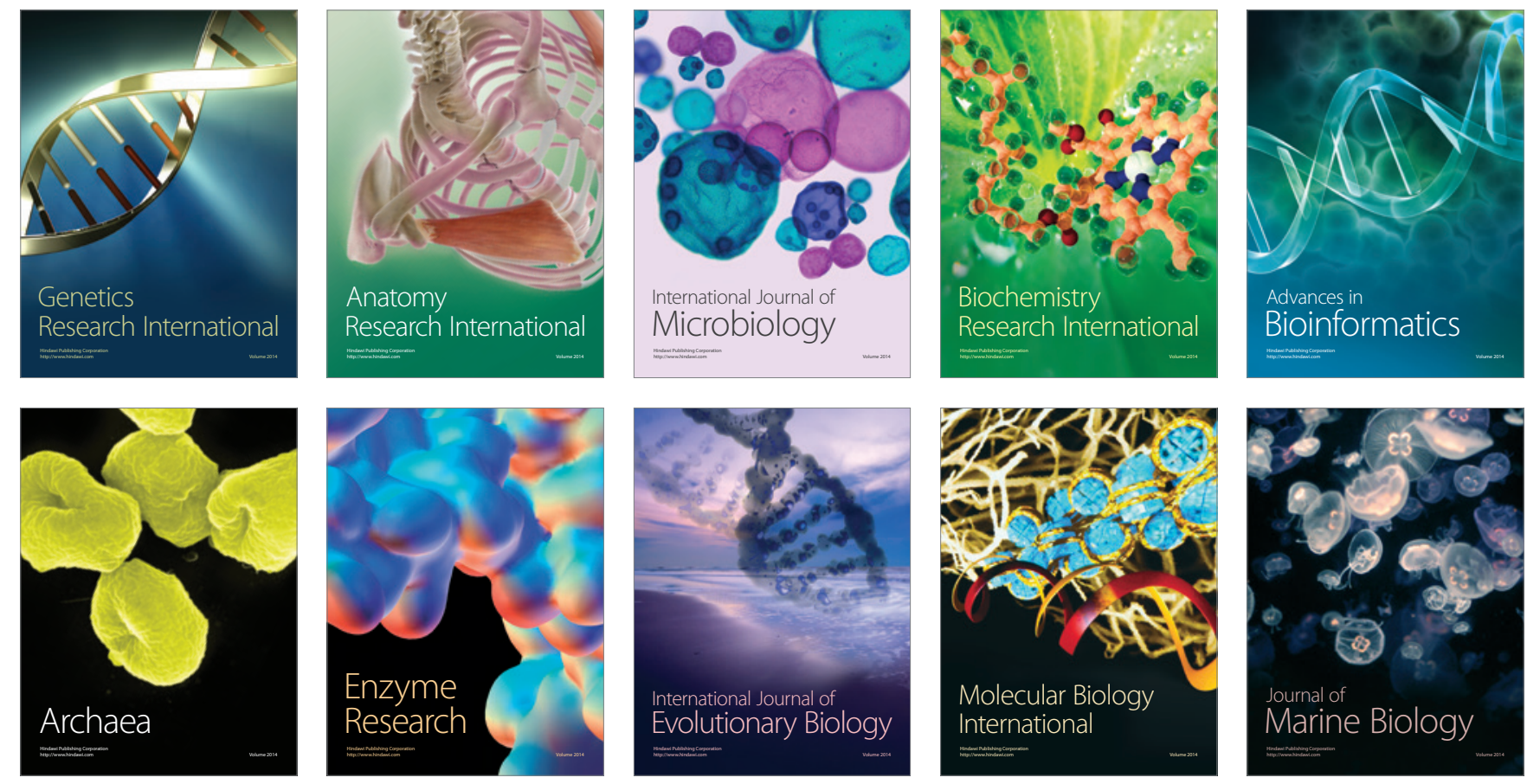